Review Article

\title{
The Effects of Oral Consumption of Honey on Key Metabolic Profiles in Adult Patients with Type 2 Diabetes Mellitus and Nondiabetic Individuals: A Systematic Review of Clinical Trials
}

\author{
Marzieh Akhbari, ${ }^{1}$ Masoumeh Jabbari, ${ }^{2}$ Mohammad Hossein Ayati $\mathbb{D}^{1,},{ }^{1,3,4}$ \\ and Nazli Namazi $\mathbb{D i}^{5}$ \\ ${ }^{1}$ School of Persian Medicine, Tehran University of Medical Sciences, Tehran, Iran \\ ${ }^{2}$ Student Research Committee, Department of Community Nutrition, Faculty of Nutrition Sciences and Food Industry, \\ Shahid Beheshti University of Medical Sciences, Tehran, Iran \\ ${ }^{3}$ Endocrinology and Metabolism Research Center, Endocrinology and Metabolism Clinical Sciences Institute, \\ Tehran University of Medical Sciences, Tehran, Iran \\ ${ }^{4}$ Department of History of Medicine, School of Traditional Medicine, Tehran University of Medical Sciences, Tehran, Iran \\ ${ }^{5}$ Diabetes Research Center, Endocrinology and Metabolism Clinical Sciences Institute, Tehran University of Medical Sciences, \\ Tehran, Iran
}

Correspondence should be addressed to Mohammad Hossein Ayati; ayatimd@gmail.com and Nazli Namazi; nazli.namazi@yahoo.com

Received 6 November 2020; Revised 27 December 2020; Accepted 7 January 2021; Published 23 January 2021

Academic Editor: Mohammad Hashem Hashempur

Copyright (c) 2021 Marzieh Akhbari et al. This is an open access article distributed under the Creative Commons Attribution License, which permits unrestricted use, distribution, and reproduction in any medium, provided the original work is properly cited.

Objectives. Although several clinical trials have revealed the beneficial effects of honey on metabolic profiles, the results are conflicting. The aim of this study was to systematically summarize the effects of oral consumption of honey on key metabolic profiles in adult patients with type 2 diabetes mellitus (T2DM) and nondiabetic individuals. Methods. In total, four electronic databases, including PubMed/Medline, Web of Science, Scopus, and Cochrane library, were searched from 2000 to 31 July 2019 to identify all English language studies that would meet the eligibility criteria. Clinical trials which have examined the effects of oral consumption of any types of honey on anthropometric indices, glycemic status, lipid profiles, and blood pressure in both diabetic and nondiabetic adult subjects were included in the study. Results. Of the 7769 possible relevant studies (including 3547 duplicates) identified in the initial search, finally, 13 clinical trials were included in the systematic review. All studies except three had a parallel design. Of 13 studies, 8 trials did not have placebo/control groups. The included studies examined the impact of oral consumption of honey on glycemic status $(n=12)$, anthropometric indices $(n=6)$, lipid profiles $(n=10)$, and blood pressure $(n=3)$. Based on the Jadad scale, 5 studies had acceptable methodological quality, and the remaining $(n=8)$ had low methodological quality. Conclusion. The current systematic review showed that oral consumption of honey might have no significant effects on the modulation of metabolic profiles in nondiabetic subjects. In addition, a high intake of honey might increase glucose levels and worsen other metabolic parameters in patients with T2DM. Due to substantial heterogeneity in study design and limited clinical trials, results, however, should be interpreted with great caution.

\section{Introduction}

According to the report published by the World Health Organization (WHO), cardiovascular diseases (CVDs) are the leading cause of death worldwide, leading to about 17.9 million deaths per year [1]. Obesity, diabetes, and dyslipidemia play the pivotal roles in the incidence of CVDs $[2,3]$. For the management of patients at high risk of CVDs, there 
are several strategies including taking medicines, lifestyle modifications, adherence to healthy diets [4], and consumption of functional foods $[5,6]$.

Functional foods can be considered as one of the useful modifiers of CVD risk factors [7]. Such types of food can either improve health status or reduce the risk of various diseases apart from providing nutritional requirements [8]. Leafy greens, berries, soy, fatty fish [9], and honey [10] are examples of functional foods.

Honey, a natural sweetener, is widely available across the world [11]. More than 300 different types of floral honey are available in the world's market places. The appearance, sensory characteristics, and the amount of biochemical components, including glucose to fructose ratio, mineral, and vitamin content of honey, vary based on botanical origin [12]. Honey is a high-carbohydrate food containing monosaccharide (glucose and fructose) and disaccharides $[12,13]$. Therefore, its effects on glycemic parameters are exceedingly important, especially for patients with diabetes mellitus (DM) and those suffering from glucose intolerance. Due to differences in physicochemical properties of honey collected from various botanical sources, the glycemic index (GI) of honey varies between 32 and 85 [12].

Honey is beyond a carbohydrate source; it contains numerous components with antioxidant, antiinflammatory, and antimicrobial characteristics including polyphenols, flavonoids, enzymes, vitamins, and trace elements $[12,14]$. In traditional medicine such as traditional Persian medicine (TPM), honey has been used as a complementary therapy for wide ranges of diseases [15] such as liver and vascular diseases. In TPM, honey is called "Angabin" and "Shahd." The type with no wax and transparent red color that is tasty and fragrance has been introduced as the best one $[16,17]$. In the conventional medicine, honey has also been used for the treatment of gastrointestinal diseases, healing of ulcers (diabetic ulcers and bedsores), skin diseases, respiratory disorders, and urinary system diseases $[15,18,19]$.

Several clinical trials have revealed the beneficial effects of honey on metabolic profiles, including lipid profiles, glycemic status, anthropometric indices, and inflammatory parameters [20-23]. However, the results are conflicting. To the best of our knowledge, no systematic review has been conducted to summarize the effects of oral consumption of honey on metabolic profiles. Accordingly, the primary objective of the present study was to examine the effects of honey on glycemic status, lipid profiles, anthropometric indices, and blood pressure in adult individuals, and the secondary objective was to compare its impacts on patients with type 2 diabetes (T2DM) and nondiabetic individuals and present findings quantitatively, if possible.

\section{Methods}

The current systematic review was designed based on the Preferred Reporting Items of Systematic Reviews and MetaAnalysis (PRISMA) statement guideline [24].

To identify relevant studies conducted on the effects of honey on metabolic parameters in adult patients with T2DM and nondiabetic individuals, the four electronic databases including PubMed/Medline, Web of Science, Scopus, and Cochrane library were searched from 2000 to July 2019 using both $\mathrm{MeSH}$ and non-MeSH terms. Notably, the search was restricted to the English language studies.

As possible relevant studies were exported into Endnote Software (version X8), two independent investigators (M.A and N.N) screened all studies based on their titles and abstracts, and those that were found to be potentially relevant were transferred to the next step in which studies were assessed based on their full-text considering eligibility criteria. Besides, to avoid missing any related studies, the reference lists of all eligible studies were hand-searched.

2.1. Eligibility Criteria. The PICO framework (P, patients/ participants; I, intervention; $\mathrm{C}$, comparison; O, outcome) was used to define the inclusion criteria. Accordingly, studies that met the following criteria were included in the review: (i) study population: subjects with T2DM or nondiabetic individuals aged 18 years and over, (ii) intervention: oral consumption of each type of honey, (iii) comparison: the control group receiving a placebo or not receiving it, (iv) outcome: at least one of the following parameters: anthropometric indices (weight, body mass index (BMI), and waist circumference (WC)), glycemic indices (fasting blood glucose (FBG), hemoglobin A1C (HbA1c), insulin, insulin resistance, and insulin sensitivity), and lipid profile (triglyceride (TG), total cholesterol (TC), low-density lipoprotein cholesterol (LDL-C), and high-density lipoprotein cholesterol (HDL-C)), and (v) duration of intervention equal or longer than 7 days. In addition, only clinical trials with either a parallel or cross-over design were considered eligible in the review.

Human studies with any other design, short-term intervention (less than 7 days), studies on children and adolescents, athletes, other types of diabetes, malignant diseases such as cancer, animal studies, in vitro studies, grey literature (conference papers, theses, and interviews), topical treatments, and studies which examined the effects of honey in combination with other materials were excluded from the study.

2.2. Data Extraction. The characteristics of the included studies were extracted by two independent reviewers (M. A and M. J). The data extraction form included the following information: the first author's last name, year of publication, location, study design, gender, mean age, sample size at baseline and the end of the trail, disease background, dosage of honey, type of honey, duration of intervention, other interventions, adjustments, outcomes, and findings.

When there were insufficient data for the included clinical trials, we contacted the corresponding author via e-mail. Unless an answer was received after three times of contact at the end of each week, it was excluded completely or for a specific parameter. Furthermore, when parameters were reported more than twice, only measurements at baseline and the end of the trial were extracted. Apart from 
honey and control groups, data obtained from the other study groups were not extracted.

2.3. Quality Assessment. To assess the quality of the eligible clinical trials, a 5-item Jadad scale was applied [25]. Jadad checklist includes three main items as follows: (i) randomization, (ii) blinding, and (iii) an account of all participants. For the first two items, two scores can be dedicated based on the provided information in the study, and the third one can obtain maximum one score. In general, any clinical trial can obtain a maximum of five scores. In the current systematic review, we considered each study with a minimum of three scores as high quality; otherwise, they were classified into the low-quality group. This section was conducted by two independent reviewers (N. N and M. A).

Any discrepancy in each of procedures was resolved by discussion or consulting with the third reviewer (MH. A) as mentioned earlier.

2.4. Data Synthesis. We found high heterogeneity in the included studies. Studies examined various types of honey and had different control groups, study designs, and study subjects. Given that this heterogeneity could not be solved by subgrouping due to limited studies with similar characteristics, we could not pool the studies to conduct a meta-analysis. Therefore, findings were reported only in a qualitative format.

\section{Results}

As depicted in Figure 1, a total of 7769 possible relevant studies (including 3547 duplicates) were identified by searching the electronic databases. After screening titles and abstracts, we found 4190 irrelevant studies excluded from the study. In the next step, full-texts of 32 articles were carefully examined. Two studies were also obtained after checking the reference lists. Twenty-one out of 34 full-text articles were excluded due to the following reasons: not clinical trial $(n=7)$, duration of intervention shorter than seven days $(n=7)$, non-English language studies $(n=3)$, and the mixture of honey with other materials $(n=4)$. In total, 13 studies were eligible and included in the qualitative synthesis.

3.1. Study Characteristics. The characteristics of 13 included clinical trials are summarized in Table 1 . The clinical trials were published between 2008 and 2019 in Asian countries $(n=10)$, European countries $(n=2)$, and the U.S.A $(n=1)$. All studies except three (cross-over) had a parallel design. All clinical trials were randomized, and 7 studies were single or double-blinded. Sample size ranged from -8 to 128 for both genders $(n=8)$, men $(n=2)$ and women $(n=3)$. They were between 18 years old and 62.8 years old. Different types of honey, including Tualang, Acacia, Rapeseed, Kelulut, and Robinia and six Greek varieties, were studied. Types of honey were not reported in some studies $(n=7)$. The effects of honey was examined in healthy $(n=4)$ and unhealthy individuals $(n=9)$, including patients with T2DM $(n=3)$. The remaining were conducted on patients with glucose intolerance $(n=3)$, high TC $(n=1)$, and overweight/obesity $(n=2)$. The duration of intervention in clinical trials with a parallel design ranged from 8 days to 12 months, and the dosage varied between $5 \mathrm{~g} /$ day and $80 \mathrm{~g} /$ day.

The included studies examined the impact of oral consumption of honey on glycemic status $(n=12)$, anthropometric indices $(n=6)$, lipid profiles $(n=10)$, and blood pressure $(n=3)$. Based on the Jadad scale, 5 studies had high methodological quality (score $\geq 3$ ), and the remaining $(n=8)$ had low methodological quality (score $<3$ ) (Table 2).

3.2. Systematic Review of Studies with Placebo Groups. In 5 clinical trials, the effects of honey on metabolic parameters were compared with placebo or control groups. In a study conducted by Yaghoobi et al., the impacts of natural honey were compared with those of sucrose in both healthy subjects and patients with high risk factors [26]. They found that $70 \mathrm{~g} /$ day honey reduced only BMI and FBS with no changes in lipid profile in healthy subjects, while it reduced TG in high risk participants. Changes in other lipid profiles and anthropometric indices were insignificant [26]. Findings of the study by Munstedt et al. revealed that a solution containing $75 \mathrm{~g}$ honey only reduced serum levels of LDL-C in women, not men. Comparison of honey with the solution containing $75 \mathrm{~g}$ glucose, and fructose showed no changes in TG and TC after 14 days [27]. According to Raatz et al., daily consumption of $50 \mathrm{~g} /$ day honey, sucrose, and high-fructose corn syrup (three study groups) increased TG and insulin concentrations in glucose-tolerant and intolerant subjects after 14 days [28]. The mentioned changes were also significant among the study groups. Rasad et al. indicated that compared to sucrose, $80 \mathrm{~g}$ /day honey solved in $250 \mathrm{~mL}$ water decreased serum levels of FBS with no changes in blood pressure in healthy young subjects after 30 days [29]. Notably, they controlled findings for age, physical activity, and some nutrient intake as confounder factors. Despland et al. compared the effects of consumption of Robinia honey, high free glucose and fructose diet, and low fructose diet (control group) in healthy normal weight men for seven days [30]. All study groups received a weight maintenance diet during the intervention. They found that diet with $25 \%$ of total energy from honey or pure fructose-glucose might slightly reduce postprandial blood glucose and insulin, while postprandial TG did not change compared to the control group [30].

Findings were classified into two groups, studies with and without control groups. In the end, the impacts of honey in patients with T2DM were also presented.

3.3. The Systematic Review Section for Studies without a Placebo Group (with a Control Group). A total of seven clinical trials with no placebo groups were included in the study. Majid et al. indicated that natural honey $(70 \mathrm{~g} /$ days for four weeks) reduced FBS, TG, and LDL-C and increased HDL-C concentrations in healthy young men compared to those continued their usual diet [31]. In their study, Sadeghi 


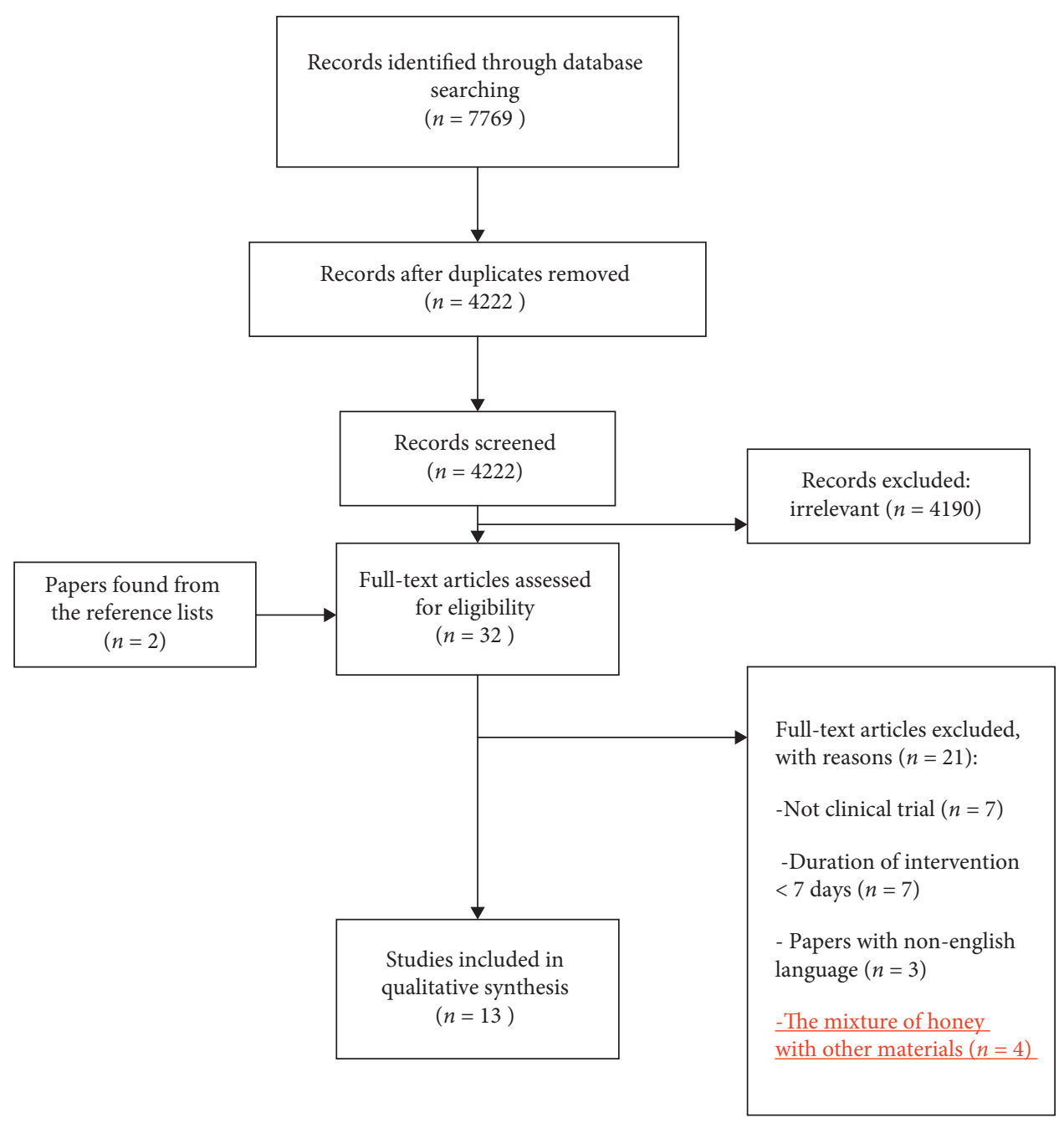

FIGURE 1: The process to reach the eligible articles.

et al. demonstrated that $50 \mathrm{~g} /$ day honey along with a weight maintenance diet increased $\mathrm{HbAlc}$ and reduced waist circumference in patients with T2DM compared to those adhered to only a weight maintenance diet after 8 weeks [32]. However, FBS, insulin, homeostatic model assessment for insulin resistance (HOMA-IR), quantitative insulin sensitivity check index (QUICKI), weight, BMI, hip circumference, and waist-to-hip ratio did not change significantly [32]. Enginyurt et al. compared the effects of three dosages of honey $(5,15$, and $25 \mathrm{~g} /$ day) with a group receiving no honey. They found that $\mathrm{HbAlc}$ was decreased in all intervention groups, but no significant changes were observed in patients with T2DM in terms of lipid profile after 4 months [33]. Mushtaq et al. demonstrated a significant reduction in serum levels of TC, LDL-C, and TG and an increase in HDL-C in groups receiving honey ( $40 \mathrm{~g} /$ day) compared to nonusers of honey in most ethnic groups after 4 weeks. They found that the effects of honey in obese subjects were higher than those in normal weight subjects [23].

Nik Hussein et al. also revealed that $20 \mathrm{~g} /$ day Tualang honey along with hormonal replacement therapy (HRT) compared to HRT alone did not have substantial effects on blood pressure, lipid profile, glucose level, BMI, and WC in postmenopausal women after 4 months [34]. Based on the report published by Rashid et al., 30 g/day Malaysian Kelulut honey did not change FBS, lipid profiles, blood pressure, and BMI in patients with IFG after 30 days as compared to control [35]. However, Bahrami et al. demonstrated that natural honey reduced bodyweight, FBS, and LDL-C/HDL-C ratio in patients with T2DM after 8 weeks compared to controls, but no changes were observed in HbAlc and lipid profiles [22]. They examined difference dosages of honey through the intervention, from $1 \mathrm{~g} / \mathrm{kg} /$ day in the first two weeks to $2.5 \mathrm{~g} /$ $\mathrm{kg} /$ day in the $7^{\text {th }}$ week and $8^{\text {th }}$ week. Notably, they adjusted findings for baseline values. Rashid et al. showed that consumption of $30 \mathrm{~g} /$ day Kelulut honey did not affect the glycemic status, lipid profiles, blood pressure, and BMI compared to those without taking honey after 1 month in patients with impaired fasting glucose [35].

In a clinical trial, the impacts of two kinds of honey (Tualang and honey cocktail) for 12 months and the results showed that honey cocktail increased BMI, while it reduced FBS. In addition, Tualang honey reduced DBP compared to honey cocktail in postmenopausal women [36]. 


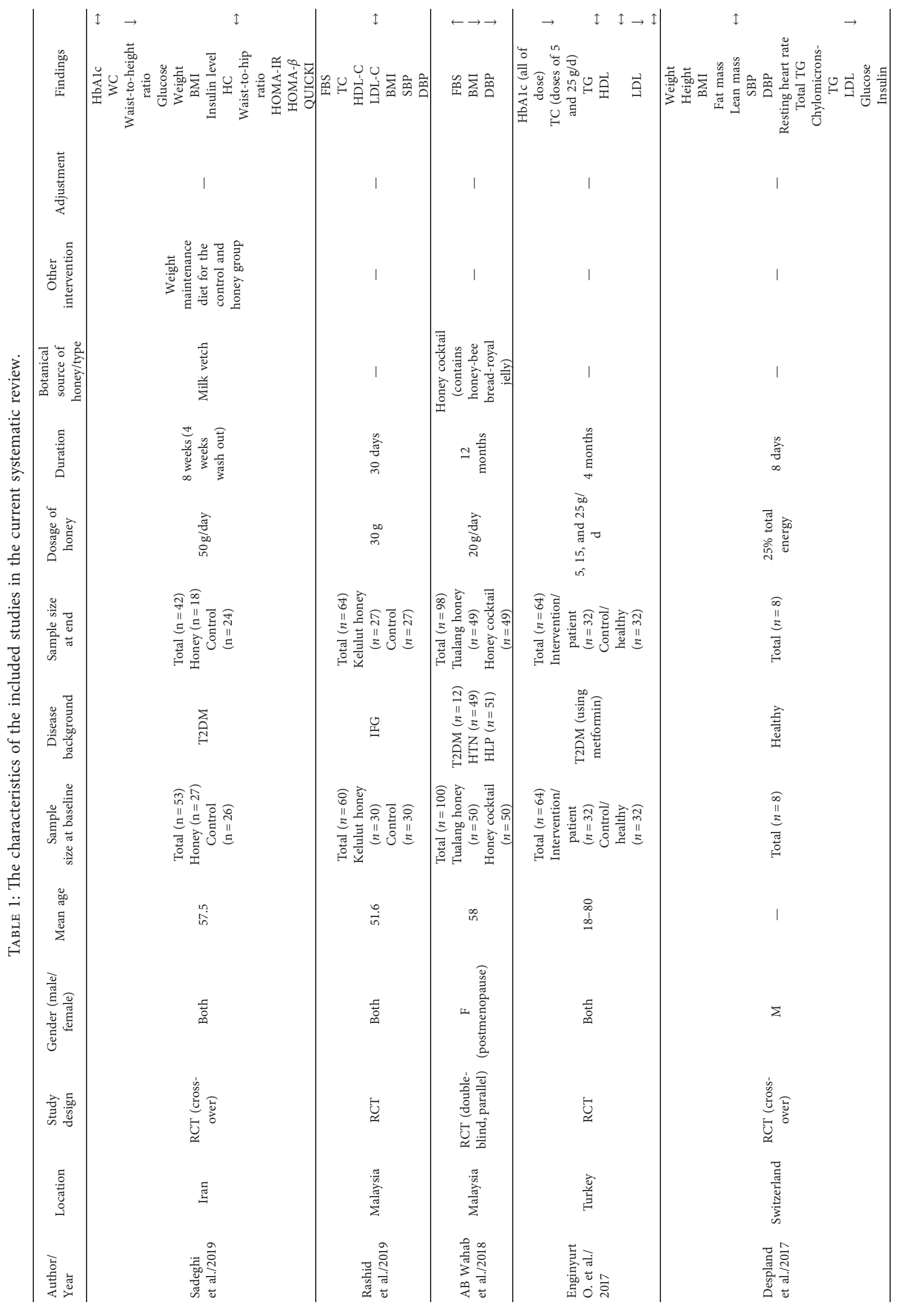




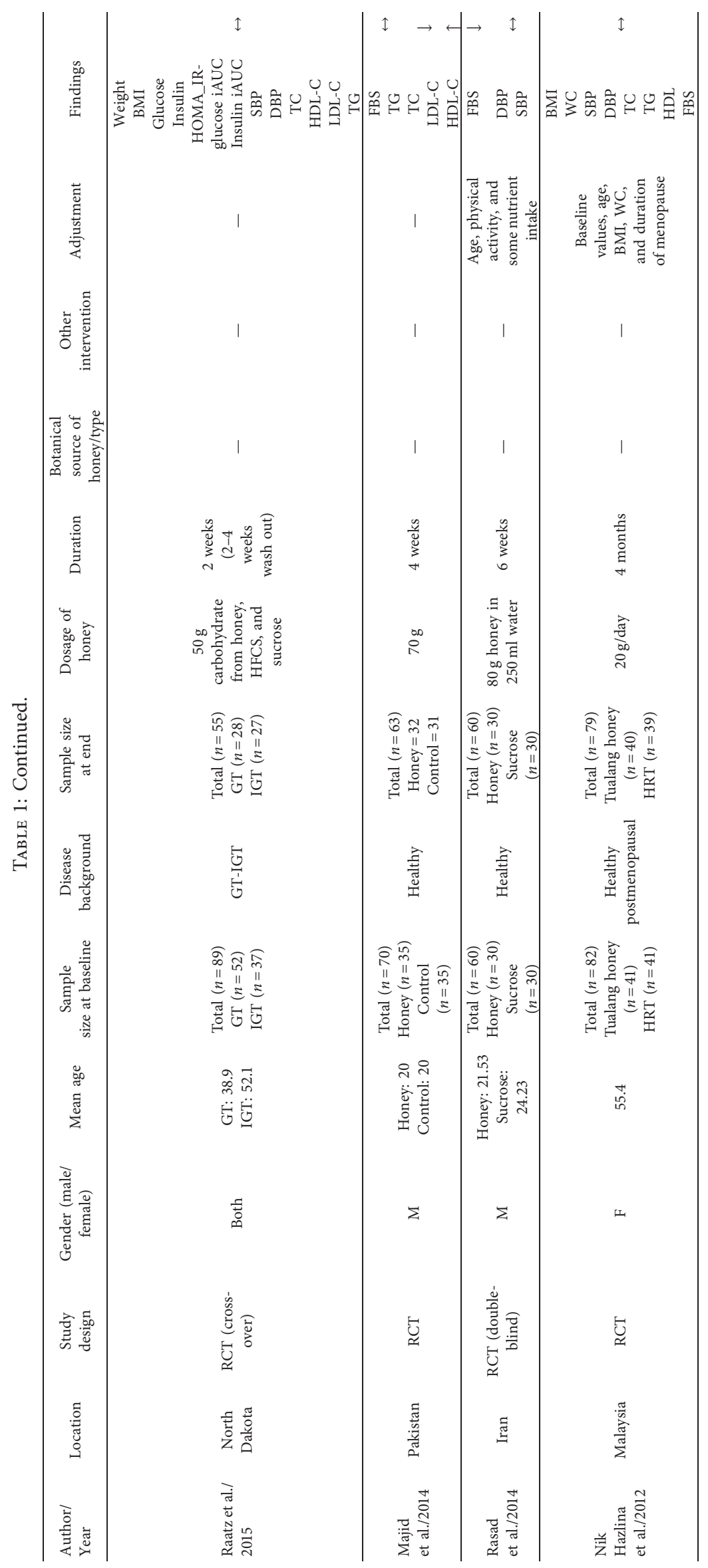




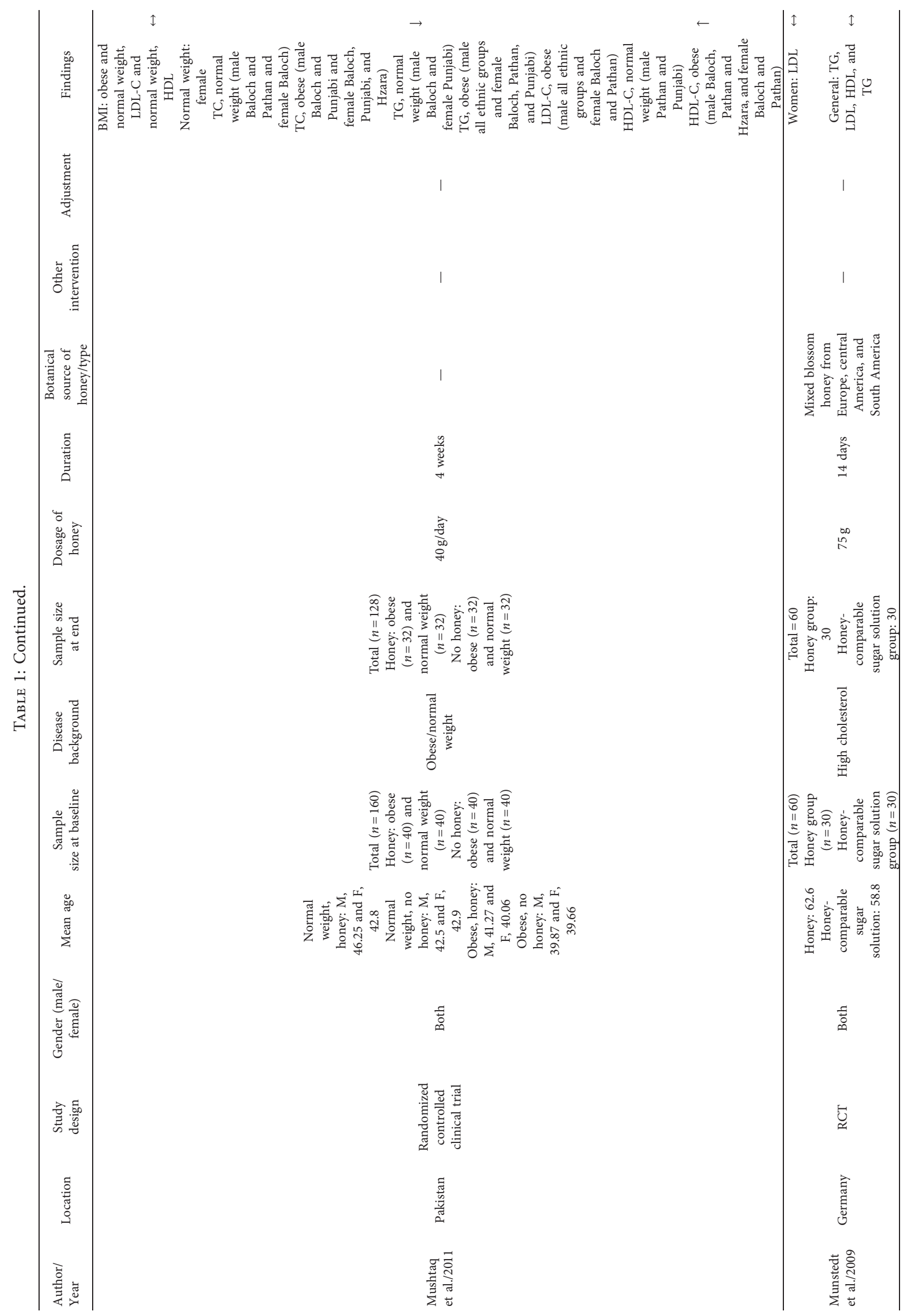




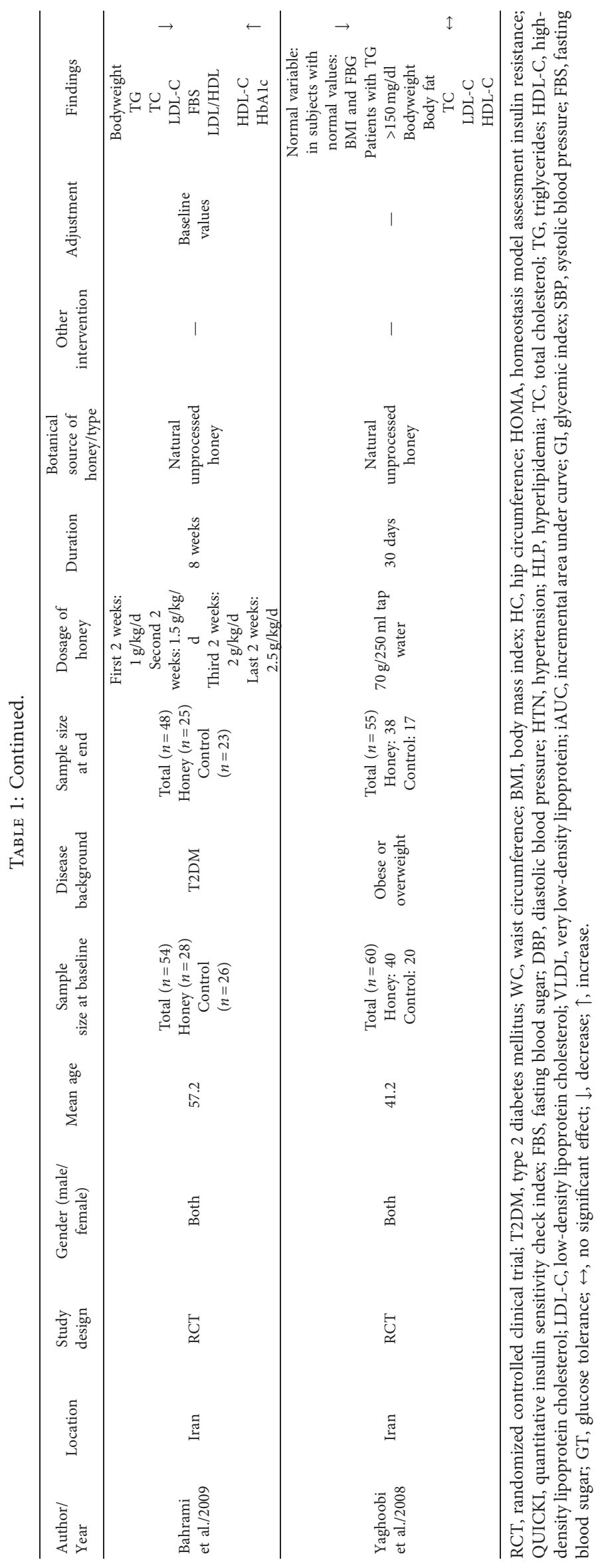


TABLE 2: The Jadad score for the included clinical trials $(n=13)$.

\begin{tabular}{|c|c|c|c|c|}
\hline Author (year) & Randomization score & Blinding score & Account of patients score & Total score \\
\hline Sadeghi et al. (2019) & 2 & 1 & 1 & 4 \\
\hline Rashid et al. (2019) & 0 & 0 & 0 & 0 \\
\hline AbWahab et al. (2018) & 2 & 2 & 1 & 5 \\
\hline Enginyurt et al. (2017) & 1 & 0 & 0 & 1 \\
\hline Despland et al. (2017) & 1 & 0 & 1 & 2 \\
\hline Raatz et al. (2015) & 1 & 0 & 1 & 2 \\
\hline Majid et al. (2014) & 2 & 1 & 1 & 4 \\
\hline Rasad et al. (2014) & 1 & 1 & 1 & 3 \\
\hline Nik Hussein et al. (2012) & 1 & 0 & 1 & 2 \\
\hline Mushtaq et al. (2011) & 0 & 0 & 0 & 0 \\
\hline Mu"nstedt et al. (2009) & 1 & 1 & 1 & 3 \\
\hline Bahrami et al. (2009) & 1 & 0 & 1 & 2 \\
\hline Yaghoobi et al. (2008) & 1 & 0 & 0 & 1 \\
\hline
\end{tabular}

3.4. The Effects of Honey in Patients with T2DM. Three clinical trials examined the effects of honey in patients with T2DM [22, 32, 33]. Two clinical trials showed a significant increase in $\mathrm{HbAlc}$ following the intake of a minimum $50 \mathrm{~g} /$ days honey for 8 weeks $[22,32]$. However, Enginyurt et al. showed that following the consumption of 5-25 g/day honey for 4 months, a reduction in HbAlc was observed [33].

\section{Discussion}

The current systematic review showed that oral consumption of honey might have no positive effects on the modulation of metabolic parameters in nondiabetic subjects. In addition, a high intake of honey might increase glucose levels and worsen other metabolic parameters in patients with T2DM. Due to substantial heterogeneity in study design and limited clinical trials, results, however, should be interpreted with great caution.

To the best of our knowledge, this is the first systematic review on the effects of oral consumption of honey on metabolic parameters in diabetic and nondiabetic individuals. Therefore, we cannot compare our findings with an earlier systematic review. We found a mixture of positive, negative, and null effects on metabolic status following the consumption of honey. This discrepancy might be due to differences in mean age, disease background, gender, BMI at baseline, dosage, duration of intervention, other intervention along with taking honey, and ethnic of participants as well as differences in types of honey obtained from various botanical sources.

In most clinical trials included in the current review, the effects of honey were not compared with those of the placebo. This point can cause considerable bias and affect both the internal and external validities of studies. Although this point is more important for subjective outcomes, it can also lead to overestimating or underestimating the real effects of the intervention for objective outcomes [37]. On the other hand, based on the Jadad scale, the most clinical trials had low methodological quality. Due to this issue, we cannot draw a fix conclusion about the effects of honey on each metabolic parameter.

Another weakness of the most included studies was related to reporting findings without controlling confounders, including baseline characteristics, total energy intake, physical activity, and BMI. The main confounder that can affect findings is total energy intake. Honey is a highnutrient functional food containing $64 \mathrm{kcal}$ in each tablespoon (about $20 \mathrm{~g}$ ). Therefore, adding honey to diet, particularly in high dosage with no replacement, no changes in daily calorie intake and physical activity can increase body weight and fat mass due to extra calorie intake. Therefore, metabolic parameters can be influenced by these changes. However, only in the two included clinical trials [30, 32], a weight maintenance diet was recommended along with honey consumption, and in most studies, findings were not reported after adjusting for such confounding factors. The importance of this issue was clarified in the study of Despland et al. They found that a diet containing 25\% of total energy intake after designing individualized weight maintenance diets did not show harmful effects on glucose and TG levels, and even, it showed reduction effects [30].

Accordingly, it seems that the negative effects of honey on metabolic parameters reported in some included clinical trials might be related to the added calorie and fructose content of honey. Some studies demonstrated that hypertriglyceridemia was associated with fructose or glucose administered in a hypercaloric diet, while no significant effects were found in a weight maintenance diet [38, 39]. Some previous studies have also showed that fructose can increase TG concentrations and stimulate hepatic de novo lipogenesis [40, 41].

Notably, the amount of fructose and fructose/glucose ratio in honey can contribute to different findings. Based on meta-analyses, postprandial TG can increase following the consumption of fructose greater than $50-60 \mathrm{~g} /$ day [42], and an increase in FBS is observed with daily dosage exceeding $100 \mathrm{~g}[42,43]$. However, the amount of fructose from honey and whole diet was reported in only two studies $[28,30]$. The average daily intake of fructose was $95 \mathrm{~g}$ in the study of Despland et al. [30]. The fructose content of honey in 
another clinical trial was also $40 \mathrm{~g} / 100 \mathrm{~g}$ of honey [28]. Fructose to glucose ratio in honey is another possible factor affecting metabolic parameters, and it differs among various types of honey. Among the included clinical trials, only Despland et al. reported the ratio, and it was 1.7 [30]. This ratio may affect both the GI of honey that is important, particularly for patients with DM and glycemic status.

It is possible that some factors, including polyphenols and other antioxidant ingredients of honey, can blunt the harmful effects of fructose on metabolic parameters. Such components are influenced by botanical sources and geographical locations [12]. Therefore, identifying the types of honey with therapeutic effects can be helpful. However, the different types of honey and their nutritional value were provided only in limited studies. Thus, we were not able to report findings based on classifications by the mentioned factors. It seems that producing honey with identified and controlled amounts of components along with adding some nutrients or materials, including probiotics and prebiotics in some cases, can be helpful, particularly for unhealthy subjects.

There were only three studies conducted on patients with T2DM $[22,32,33]$. Although this number is insufficient to conclude how much honey can be allowed for diabetic patients, we can conclude that it is not necessary to delete honey completely from diabetic diets and only restriction and to replace with other carbohydrate sources along with the maintenance of total energy intake is sufficient. One out of three studies conducted on diabetes examined different dosages of honey (5-25 g/days) [33]. The results showed that these dosages not only had no negative effect on metabolic parameters but also reduced HbA1C. However, higher dosage (50 g/day) increased HbA1c in patients with T2DM [32].

It is not fully understood by which mechanisms honey can affect metabolic profiles. However, some potential pathways are suggested. The main possible mechanisms are related to the ingredients of honey, particularly polyphones and flavonoids with anti-inflammatory and antioxidant properties. Through the suppression of inflammatory pathways, reducing free radicals, and helping to rebalance oxidant and antioxidant factors, modulation of glycemic status, lipid profiles, and other metabolic factors can be occur. Other mechanisms are reduction in body weight, increased satiety, delay in gastric emptying, and modulation of appetite hormones including neuropeptide $\mathrm{Y}$ and ghrelin due to phenolic components and oligosaccharides content [32], affecting C-peptide, stimulating beta cells of the pancreas due to antioxidant components [35], an increase in uptake of hepatic glucose and glycogen synthesis and storage due to fructose [33].

However, this study has some limitations that should be addressed. They are as follows: (i) due to high heterogeneity in the methodology of the included clinical trials and limited studies with similar characteristics, we were not able to do a meta-analysis, (ii) studies conducted on other metabolic profiles such as inflammatory and antioxidant parameters were not included, (iii) we could not determine cutoff points for dosage of honey with no negative effect on metabolic parameters due to high heterogeneity. However, doing a systematic review on this topic for the first time, examining the quality of studies, providing results separately for both diabetic and nondiabetic subjects were the strengths of this systematic review.

\section{Conclusion}

The current systematic review revealed that oral consumption of honey might have no beneficial effects on the modulation of metabolic status in nondiabetic subjects. Even high intake of honey might increase glucose levels and worsen other metabolic parameters in patients with T2DM. Due to substantial heterogeneity in study design, low quality in most clinical trials, and limited included studies, results, however, should be interpreted with great caution. More high quality randomized controlled clinical trials on different types of honey (with determined physicochemical properties) with various dosages and longer duration of the intervention are necessary to clarify the effects of honey in diabetic and nondiabetic individuals.

\section{Data Availability}

No data were used to support this study.

\section{Conflicts of Interest}

The authors declare that they have no conflicts of interest.

\section{Authors' Contributions}

N. N. and M. H. A. designed and directed the project. N. N. and M. A. provided search strategies and collection of publications in Endnote. M. A. and N. N. screened publications. M. J. and M. A. extracted data. M. A. and N. N. assessed the quality of publications. M. H. A. examined results and interpreted findings. All authors checked and confirmed the draft of the manuscript.

\section{References}

[1] World Health Organization, "Non-communicable diseases," 2018, https://www.who.int/news-room/fact-sheets/detail/ noncommunicable-diseases.

[2] C. J. Lavie, A. De Schutter, P. Parto et al., "Obesity and prevalence of cardiovascular diseases and prognosis-the obesity paradox updated," Progress in Cardiovascular Diseases, vol. 58, no. 5, pp. 537-547, 2016.

[3] Z.-J. Yang, J. Liu, J.-P. Ge, L. Chen, Z.-G. Zhao, and W.-Y. Yang, "Prevalence of cardiovascular disease risk factor in the Chinese population: the 2007-2008 China national diabetes and metabolic disorders study," European Heart Journal, vol. 33, no. 2, pp. 213-220, 2012.

[4] C. P. Cannon, "Cardiovascular disease and modifiable cardiometabolic risk factors," Clinical Cornerstone, vol. 8, no. 3, pp. 11-28, 2007.

[5] L. Brown, H. Poudyal, and S. K. Panchal, "Functional foods as potential therapeutic options for metabolic syndrome," Obesity Reviews, vol. 16, no. 11, pp. 914-941, 2015.

[6] E. M. Alissa and G. A. Ferns, "Functional foods and nutraceuticals in the primary prevention of cardiovascular 
diseases," Journal of Nutrition and Metabolism, vol. 2012, Article ID 569486, 16 pages, 2012.

[7] C. Johnston, "Functional foods as modifiers of cardiovascular disease," American Journal of Lifestyle Medicine, vol. 3, no. 1, pp. 39S-43S, 2009.

[8] A. M. Abdel-Salam, "Functional foods: hopefulness to good health," American Journal of Food Technology, vol. 5, no. 2, pp. 86-99, 2010.

[9] M. B. Roberfroid, "Concepts and strategy of functional food science: the European perspective," The American Journal of Clinical Nutrition, vol. 71, no. 6, pp. 1660S-1664S, 2000.

[10] S. Bogdanov, "Honey as nutrient and functional food," Proteins, vol. 1100, pp. 1400-2700, 2012.

[11] M. Krpan, K. Marković, G. Šarić, B. Skoko, M. Hruškar, and N. Vahčić, "Antioxidant activities and total phenolics of Acacia honey," Czech Journal of Food Sciences, vol. 27, no. Special issue 1, pp. S245-S247, 2009.

[12] S. Bogdanov, T. Jurendic, R. Sieber, and P. Gallmann, "Honey for nutrition and health: a review," Journal of the American College of Nutrition, vol. 27, no. 6, pp. 677-689, 2008.

[13] J. M. Alvarez-Suarez, S. Tulipani, S. Romandini, E. Bertoli, and M. Battino, "Contribution of honey in nutrition and human health: a review," Mediterranean Journal of Nutrition and Metabolism, vol. 3, no. 1, pp. 15-23, 2010.

[14] V. R. Pasupuleti, L. Sammugam, N. Ramesh, and S. H. Gan, "Honey, propolis, and royal jelly: a comprehensive review of their biological actions and health benefits," Oxidative Medicine and Cellular Longevity, vol. 2017, Article ID 1259510, 21 pages, 2017.

[15] N. H. A. El-Soud, "Honey between traditional uses and recent medicine," Macedonian Journal of Medical Sciences, vol. 5, no. 2, pp. 205-214, 2012.

[16] M. AghiliKhorasani, Makhzan-al-Adviah, pp. 545-563, Sabzara publications, Tehran, Iran, 2011.

[17] M. Moemen, Tohfeh-al-moemenin, pp. 592-593, Noorevahy publication, Ghom, 2011.

[18] T. Eteraf-Oskouei and M. Najafi, "Traditional and modern uses of natural honey in human diseases: a review," Iranian Journal of Basic Medical Sciences, vol. 16, no. 6, pp. 731-742, 2013.

[19] B. Medhi, A. Puri, S. Upadhyay, and L. Kaman, "Topical application of honey in the treatment of wound healing: a metaanalysis," JK Science, vol. 10, no. 4, pp. 166-169, 2008.

[20] N. S. Al-Waili, "Natural honey lowers plasma glucose, C-reactive protein, homocysteine, and blood lipids in healthy, diabetic, and hyperlipidemic subjects: comparison with dextrose and sucrose," Journal of Medicinal Food, vol. 7, no. 1, pp. 100-107, 2004.

[21] N. M. Arani, Z. Emam-Djomeh, H. Tavakolipour, R. Sharafati-Chaleshtori, A. Soleimani, and Z. Asemi, "The effects of probiotic honey consumption on metabolic status in patients with diabetic nephropathy: a randomized, doubleblind, controlled trial," Probiotics and Antimicrobial Proteins, vol. 11, pp. 1195-1201, 2019.

[22] M. Bahrami, A. Ataie-Jafari, S. Hosseini, M. H. Foruzanfar, M. Rahmani, and M. Pajouhi, "Effects of natural honey consumption in diabetic patients: an 8-week randomized clinical trial," International Journal of Food Sciences and Nutrition, vol. 60, no. 7, pp. 618-626, 2009.

[23] R. Mushtaq, R. Mushtaq, and Z. T. Khan, "Effects of natural honey on lipid profile and body weight in normal weight and obese adults: a randomized clinical trial," Pakistan Journal of Zoology, vol. 43, no. 1, pp. 161-169, 2011.
[24] D. Moher, A. Liberati, J. Tetzlaff, and D. Altman, "Preferred reporting items of systematic review and meta-analyses: the PRISMA statement," Deutsche Medizinische Wochenschrift, vol. 136, no. 15, 2011.

[25] H. D. Clark, G. A. Wells, C. Huët et al., "Assessing the quality of randomized trials," Controlled Clinical Trials, vol. 20, no. 5, pp. 448-452, 1999.

[26] N. Yaghoobi, N. Al-Waili, M. Ghayour-Mobarhan et al., "Natural honey and cardiovascular risk factors; effects on blood glucose, cholesterol, triacylglycerole, CRP, and body weight compared with sucrose," The Scientific World Journal, vol. 8, pp. 463-469, Article ID 961837, 2008.

[27] K. Münstedt, S. Hoffmann, A. Hauenschild, M. Bülte, R. Von Georgi, and A. Hackethal, "Effect of honey on serum cholesterol and lipid values," Journal of Medicinal Food, vol. 12, no. 3, pp. 624-628, 2009.

[28] S. K. Raatz, L. K. Johnson, and M. J. Picklo, "Consumption of honey, sucrose, and high-fructose corn syrup produces similar metabolic effects in glucose-tolerant and -intolerant individuals," The Journal of Nutrition, vol. 145, no. 10, pp. 2265-2272, 2015.

[29] H. Rasad, A. Dashtabi, M. Khansari et al., "The effect of honey consumption compared with sucrose on blood pressure and fasting blood glucose in healthy young subjects," Global Journal of Medicine Research and Studies, vol. 1, no. 4, pp. 117-121, 2014.

[30] C. Despland, B. Walther, C. Kast et al., "A randomizedcontrolled clinical trial of high fructose diets from either Robinia honey or free fructose and glucose in healthy normal weight males," Clinical Nutrition ESPEN, vol. 19, pp. 16-22, 2017.

[31] M. Majid, M. A. Younis, A. K. Naveed, M. U. Shah, Z. Azeem, and S. H. Tirmizi, "Effects of natural honey on blood glucose and lipid profile in young healthy Pakistani males," Journal of Ayub Medical College Abbottabad, vol. 25, no. 3-4, pp. 44-47, 2013.

[32] F. Sadeghi, S. Salehi, A. Kohanmoo, and M. Akhlaghi, "Effect of natural honey on glycemic control and anthropometric measures of patients with type 2 diabetes: a randomized controlled crossover trial," International Journal of Preventive Medicine, vol. 10, no. 3, pp. 1-10, 2019.

[33] O. Enginyurt, L. Cakir, A. Karatas et al., "The role of pure honey in the treatment of diabetes mellitus," Biomedical Research, vol. 28, no. 7, pp. 3305-3312, 2017.

[34] N. H. N. Hussain, S. A. Sulaiman, I. Hassan et al., "Randomized controlled trial on the effects of tualang honey and hormonal replacement therapy (HRT) on cardiovascular risk factors, hormonal profiles and bone density among postmenopausal women: a pilot study," Journal of Food Research, vol. 1, no. 2, pp. 171-189, 2012.

[35] M. R. Rashid, K. N. Nor Aripin, F. B. Syed Mohideen et al., "The effect of Kelulut honey on fasting blood glucose and metabolic parameters in patients with impaired fasting glucose," Journal of Nutrition and Metabolism, vol. 2019, Article ID 3176018, 7 pages, 2019.

[36] S. Z. Ab Wahab, N. H. Nik Hussain, R. Zakaria et al., "Longterm effects of honey on cardiovascular parameters and anthropometric measurements of postmenopausal women," Complementary Therapies in Medicine, vol. 41, pp. 154-160, 2018.

[37] A. Hróbjartsson, T. J. Kaptchuk, and F. G. Miller, "Placebo effect studies are susceptible to response bias and to other types of biases," Journal of Clinical Epidemiology, vol. 64, no. 11, pp. 1223-1229, 2011. 
[38] R. D. Johnston, M. C. Stephenson, H. Crossland et al., "No difference between high-fructose and high-glucose diets on liver triacylglycerol or biochemistry in healthy overweight men," Gastroenterology, vol. 145, no. 5, pp. 1016-1025, 2013.

[39] E. T. N. Sock, K.-A. Lê, M. Ith, R. Kreis, C. Boesch, and L. Tappy, "Effects of a short-term overfeeding with fructose or glucose in healthy young males," British Journal of Nutrition, vol. 103, no. 7, pp. 939-943, 2010.

[40] L. Egli, V. Lecoultre, F. Theytaz et al., "Exercise prevents fructose-induced hypertriglyceridemia in healthy young subjects," Diabetes, vol. 62, no. 7, pp. 2259-2265, 2013.

[41] J.-M. Schwarz, S. M. Noworolski, M. J. Wen et al., "Effect of a high-fructose weight-maintaining diet on lipogenesis and liver fat," The Journal of Clinical Endocrinology \& Metabolism, vol. 100, no. 6, pp. 2434-2442, 2015.

[42] G. Livesey and R. Taylor, "Fructose consumption and consequences for glycation, plasma triacylglycerol, and body weight: meta-analyses and meta-regression models of intervention studies," The American Journal of Clinical Nutrition, vol. 88, no. 5, pp. 1419-1437, 2008.

[43] J. L. Sievenpiper, A. J. Carleton, S. Chatha et al., "Heterogeneous effects of fructose on blood lipids in individuals with type 2 diabetes: systematic review and meta-analysis of experimental trials in humans," Diabetes Care, vol. 32, no. 10, pp. 1930-1937, 2009. 